\title{
Spontaneous and Deterministic Three-Dimensional Curling of Pre-Strained Elastomeric Bi-Strips
}

\section{Citation}

Huang, Jiangshui, Jia Liu, Benedikt Kroll, Katia Bertoldi, and David R. Clarke. 2012. Spontaneous and deterministic three-dimensional curling of pre-strained elastomeric bi-strips. Soft Matter 8(23): 6291-6300.

\section{Published Version}

doi:10.1039/c2sm25278c

\section{Permanent link}

http://nrs.harvard.edu/urn-3:HUL.InstRepos:9177225

\section{Terms of Use}

This article was downloaded from Harvard University's DASH repository, and is made available under the terms and conditions applicable to Open Access Policy Articles, as set forth at http:// nrs.harvard.edu/urn-3:HUL.InstRepos:dash.current.terms-of-use\#OAP

\section{Share Your Story}

The Harvard community has made this article openly available.

Please share how this access benefits you. Submit a story.

\section{Accessibility}




\title{
SPONTANEOUS AND DETERMINISTIC THREE-DIMENSIONAL CURLING OF PRE-STRAINED ELASTOMERIC BI-STRIPS
}

\author{
Jiangshui Huang, Jia Liu, Benedikt Kroll, Katia Bertoldi and David R. Clarke * \\ School of Engineering and Applied Sciences \\ Harvard University, Cambridge, MA 02138
}

\begin{abstract}
Three dimensional curls ("hemi-helices") consisting of multiple, periodic and alternating helical sections of opposite chiralities, separated by perversions, are one of a variety of complex shapes that can be produced by a simple generic process consisting of pre-straining one elastomeric strip, joining it side-by-side to another and then releasing the bi-strip. The initial wavelength of the hemi-helix and the number of perversions are determined by the strip crosssection, the constitutive behavior of the elastomer and the value of the pre-strain. The hemihelix has no net twist. Topologically, the perversions separate regions of the hemi-helix deforming principally by bending from those where twisting dominates.
\end{abstract}

\section{INTRODUCTION}

Nature abounds with complex three-dimensional morphologies ${ }^{1,2}$, many of which can be reproduced by simple growth rules, for instance by diffusion limited aggregation ${ }^{3}$ and cellular automata ${ }^{4}$. Other complex shapes can be generated in initially flat sheets or ribbons by simple operations. For instance, the Mobius strip can be produced by taking a long strip of material, twisting one end with respect to the other and then joining the two ends of the twisted strip. Moreover, Sharon and Efrati ${ }^{5}$ have demonstrated that complex shapes can be produced by a differential swelling operation and recently Savin et al. ${ }^{6}$ have shown that the looped pattern of the gut can be reproduced by a combination of growth and bending of a tube 
along its length but constrained at its two ends. In this paper we describe the formation a hitherto unreported three-dimensional shape -- which we term a hemi-helix -- produced by another simple set of generic operations on a 1D system but without any volume change, plastic strain, or differential swelling: elongating one straight strip, joining it side-by-side to a second, flat and unstrained strip and then releasing the bi-strip formed. The hemi-helix shape consists of periodic and alternating helical sections of opposite chiralities, separated by "perversion defects", is free-standing and is mechanically stable. Furthermore, its initial wavelength is determined by the initially applied pre-stretch. An example is shown in figure 1 as a strained bi-strip elastomer is released by incrementally decreasing the distance between its ends. Unlike the shapes produced in the above referenced works in the literature, the formation of the hemi-helix is associated with a twist component of deformation. We will show through simulation that the hemi-helix shape has a higher elastic energy than the simple helix and it is trapped in the higher energy state because of the twist deformation locked in by perversion defects.

Among other shapes, helices are ubiquitous in nature and the study of their formation and properties has attracted considerable interest for many years. For instance, it has been found that a rich phenomenology of helical shapes can be obtained in narrow bilayered ribbons depending on the orientation and width of the strips 7,8 . In twist-nematic elastomers, transitions between helicoids and helical ribbons has been reported as the width increases ${ }^{9}$. Moreover, the degree of twist and the pitch of ribbons in bilayers of Gemini surfactants have been successfully tuned introducing opposite-handed chiral counterions in various proportions 10. Inversion of chirality is observed in some helices. For instance, plant tendrils can switch from a left-handed helix to a right-handed one (or vice-versa) at some point in their growth as originally discussed by Darwin. (An excellent account of the history of understanding perversions in tendrils is given by McMillen and Goriely ${ }^{11}$ ). As Darwin proposed and has been discussed more recently in topological terms ${ }^{12}$, once the growing tendril has attached to a support, it's ends are fixed and so to minimize the possibility of breaking by continued twisting, a perversion forms to create equal left and right-handed segments and the net twist is conserved. Similarly, bacteria can change direction of motion propagating a right-handed helix into a left-handed helix, thus creating a perversion ${ }^{13}$. Most recently, in the chemical literature, peptide molecules have been synthesized that switch from one chirality to another along their 
length ${ }^{14}$. Although helical structures with one perversion have been reported on several occasions in the biological and plant literature as mentioned above, three dimensional structures with several periodically arranged perversions have not been. In the area of mechanics, numerical studies of the buckling of stretched slender, long rods with an initial curvature have revealed that a single perversion can form when they are compressed ${ }^{11,13}$ but there have been no previous reports of multiple perversions that we are aware of. Thus, not only is the formation of hemi-helices a new and unexpected morphological response to a very simple generic pre-straining rule applied to a bi-strip but also the creation of numerous perversions, periodically arranged along the bi-strip provides an opportunity to investigate aspects of perversions themselves.

In this contribution, we describe the formation of hemi-helices, the dependence of their characteristic features, such as initial wavelength and number of perversions on the values of the pre-strain and geometrical cross-section, and show that all the experimental observations can be reproduced through simulation using the well-established Gent constitutive law to represent the deformation behavior of elastomeric materials. We also demonstrate by simulation that the hemi-helix is a higher energy configuration than a simple helix and is trapped in that state by the presence of the perversions. Finally, we show that the formation of a hemi-helix does not depend on the non-linearity of the elastomer and linear elastic materials can be expected to form hemi-helices if they can be pre-strained sufficiently without breaking.

\section{OBSERVATIONS}

The sequence of operations applied to the two strips of elastomer of different initial length is shown schematically in figure 2. In the first step, the shorter, red strip, length $L^{\prime}$ is stretched by pulling on its ends to be equal in length to the longer blue-green strip, length, $L$. This operation produces an elongational pre-strain defined as $\chi=\left(L-L^{\prime}\right) / L^{\prime}$, in the red strip. While still stretched, the red strip is then glued along its narrow edge to an unstrained strip of the same elastomeric material, but dyed blue-green, and of the same length as the elongated strip, $L$, along its narrow edge. The glue used is another silicone rubber product that has an elastic modulus reported to be the same as that of the elastomer strips. At this stage, the bistrip is flat and no curvature is observed. Then, in the third step, the force stretching the red 
strip is gradually released. As the force is released, the bi-strip distorts out of plane, twisting and bending to produce the complex helical-like shape shown in the sequence of successive photographs in Figure 1 (a). We refer to this new geometrical shape as a hemi-helix. A line having a hemi-helical shape can be described mathematically as presented in Appendix One.

In this particular example, the initial length of the bi-strip was $L=50 \mathrm{~cm}$ and the length of the bi-strip recorded at the successive images is shown at the right hand side of figure 1. Evidently, out-of-plane distortions develop very early while their amplitudes increase as unloading continues. Inspection of the bi-strip reveals another characteristic feature; irrespective of the value of the pre-strain, $\chi$, it consists of alternating helical sections of opposite chiralities. The junctions between these alternating chiralities - sometimes referred to as perversions ${ }^{11}$ - develop early in the unloading process but the number of them, $N$, does not change with continuing unloading, although the average buckling wavelength, as well as the length between the ends of the strip, decreases as the force holding the ends is decreased. For the bi-strip shown in Fig. 1 there are eleven, arrowed in figure 1 (a). (We note that spacing of the perversions is not perfectly periodic and the wavelengths along the hemi-helix -vary a little but attribute this to random, local imperfections in the bi-strip. The observations, including the local variations in wavelength are, however, fully reproducible in any one bi-strip; the same bistrip can be released to form the hemi-helix, stretched back to being a straight bi-strip, released again innumerable times and each time the initial wavelength of the hemi-helix is the same and the same number of perversions form. We also observed the same number of perversions, when multiple bi-strips of the same dimensions and the same value of the pre-stretch are made. Also, although the cross sections of the two strips are not equal (the pre-stretched one is smaller due to Poisson's contraction), the occurrence of hemi-helix is found not to be affected by this, as confirmed by repeating the experiment using two strips with the same cross-sections after pre-straining.

The spatial separation into regions of predominately twisting and bending can be clearly seen in the micro-computerized tomography (micro-CT) image of a similar hemi-helix shown in Figure 3. The image reveals that the bi-strip is locally bent about an axis approximately perpendicular to the line originally joining the ends of the bi-strip, with little twist but substantial bending, at the perversions, marked by the dashed vertical lines. In contrast, 
between the perversions, the strip is twisted about the long axis of the hemi-helix with a small degree of bending. This suggests that the perversions form so as to produce alternating regions of large twist and small bending separated from regions, where the perversion is geometrically located, of small twist and large bending. This spatial partitioning of bending and twisting distortions is analogous to a form of phase separation or ordering in which the perversions are the equivalents of walls or interfaces, in this case, diffuse interfaces. The most direct analogy is perhaps the formation of walls that separate regions of different order parameter ${ }^{15} 16$, the simplest case, for instance, being twin boundaries. If the perversions are considered as diffuse interfaces separating regions of opposite chiralities, then it can be anticipated that the number will be determined by the minimization of the sum of the perversion interface energies, the stretching, bending and twisting energies, including gradient energy terms.

While the photographs in figure 1 were obtained by holding the ends of the bi-strip and slowly bringing them together along a straight line without twisting, the same shape, complete with the same number of perversions, is obtained under a variety of boundary conditions, namely if: (i) the ends of the stretched strips are suddenly released or (ii) the bi-strip is released under steadily decreasing load, or (iii) one end is free to rotate and move while the other is fixed. In the third case, the free end is observed not to rotate even though it is free to do so. This is shown in a video recording in the Supplemental Materials of the release of a stretched bi-strip whose ends are free to rotate.

Although the formation of a hemi-helix is always observed during the release process, it is not apparently the uniquely stable configuration. For instance, the twisted bi-strip of figure 1 (a) and 1 (b) can have a shorter final length and a topologically simpler structure if, as the ends are gradually brought together, one end is manually rotated relative to the other. Then, the bistrip adopts a tightly packed, simple linear helix in the fully released state as illustrated in figure 1 (c), recorded at the same magnification as the hemi-helix in figure 1 (b). This structure is distinctly different as it does not express any perversions; there is little twist and the helical wavelength as well as the final length is consistently smaller although the radius of curvature is, within experimental uncertainty, the same. Finally, it is also interesting to observe that in the fully released state where the deformation is dominated by bending with little remaining twist, both the hemi-helix and helix are characterized by the same major radius of the coils viewed 
along the long axis of the hemi-helix. This can be seen by comparing the diameter in the images, recorded at the same magnification, of figure $1(b)$ and 1 (c). Indeed, the radius of the hemi-helix and the helix are both dependent on the value of the elongational pre-strain. Measurements of this radius are presented in figure 4 together with an analytical solution for the curvature developed in Appendix Two.

The dynamics of the hemi-helix when the ends are rotated are also notable. When one end of the hemi-helix is held fixed and the other rotated uniformly at a constant rate to convert to a regular helix, it does not un-twist uniformly. Instead, it does so by a series of sudden "snapthrough" rotations, each of which abruptly removes one perversion at a time. Concurrently, there is a spatial re-organization along the strip as the hemi-helical wavelength adjusts to a new value and the spacing between the remaining perversions increases. The dynamics of the process following this "snap-through" rotation are as if the positions of the perversions rearrange because they repel one another.

To investigate the dependency of the initial buckling wavelength of the hemi-helix on the pre-strain and the thickness and width of the strips, we fabricated and tested a set of bistrip samples where we systematically changed both the pre-strain and the cross-section. The initial buckling wavelength, defined as $\lambda_{O}=2 L / N$, developed as the ends of the bi-strip were gradually released is found to depend on the pre-strain, $\chi$, as well as the geometrical width, $w$, and thickness, $h$, of the individual strips, defined in figure 2, as shown in figure 5 (a) and 5 (b), respectively. The initial average wavelength varies as a power law of the pre-strain according to $(\chi)^{-1 / 3}$. The variation with the width and thickness are slightly different, $w^{2 / 5}$ and $h^{3 / 5}$, respectively. All the data obtained fits a linear relationship between the wavelength and a length scaling parameter $w^{2 / 5} h^{3 / 5} \chi^{-1 / 3}$ shown in figure 5 (c). The physical basis for these particular scaling dependences on the individual width and thickness parameters is not known although the last scaling relation follows from dimensional arguments alone. Nevertheless, while we do not have analytical expressions that lead to these parametric dependencies they are reproduced by the simulations as will be described in the following section. 


\section{MODELINGANDANALYSIS}

Conceptually, the deformation observed on releasing the ends of the stretched bi-strip can be considered as an unusual form of buckling instability with the onset of out-of-plane twisting and bending being symmetry breaking: the twist can form in either a clockwise or anticlockwise manner at each end even though the net twist must remain zero if the ends do not rotate. At the onset of the release process, the bi-strip is subject to a uniaxial state of deformation; the blue-green strip is under compression while red strip is under tension. During the continued release, the tensile stress in the red strip decreases while the blue-green strip is compressed further and at some strain, relative to the initial, pre-stretched state, bifurcation occurs and the bi-strip buckles. This process can be seen in the first sequences of images in figure 1 (a). In essence, the shortening of the red strip provides the driving force for the instability since to accommodate the shortening of the red strip, the blue-green strip is progressively compressed. Therefore at a critical decrease in length, the bi-strip will begin to buckle out of plane. Formally, this process is analogous to the buckling of a strip attached to a foundation ${ }^{17}$ although the foundation in this case is a narrow strip of the same material rather than a large, solid substrate that is usually the subject of analysis. To study the formation of the hemi-helix and the conditions under which it forms, the combination of twisting, compression and bending, together with the highly nonlinear constitutive behavior of elastomer need to be included. To do this, we have used numerical simulations employing finite element modeling to investigate both the buckling behavior and the post-buckling response of the bi-strip. Before describing these simulations in detail, the constitutive behavior of the elastomer is first presented.

\section{Material constitutive behavior.}

To characterize the constitutive response of the elastomer for use in the simulations, uniaxial tensile stress-strain tests were conducted on the elastomer. The tests show that the material exhibits a large strain elastic behavior typical of elastomers with significant stiffening (see Fig. 6). To capture the observed deformation response we modeled the material as a hyper-elastic solid ${ }^{18}$, and computed the stresses and elastic energies using the nearly- 
incompressible Gent model ${ }^{19}$, typically used for simulating elastomers. The basis of this phenomenological model is that it captures the limiting molecular chain extensibility at the molecular level that leads to the deformation-induced stiffening of the elastomer.

Formally, the deformation is described in terms of a local deformation gradient, $\mathbf{F}=\frac{\partial \mathbf{x}}{\partial \mathbf{X}}$, mapping a point in the material from the reference position $\mathbf{X}$ to its current location $\mathbf{x}$. We denote $J$ as its determinant, $J=\operatorname{det}(\mathbf{F})$. For an isotropic hyperelastic material, the strain energy, $\mathbf{W}$, is a function of the invariants of the tensor $\mathbf{B}=\mathbf{F F}^{\mathbf{T}}$, the left Cauchy-Green tensor:

$$
W=W\left(I_{1}, I_{2}, I_{3}\right)
$$

where

$$
I_{1}=\operatorname{tr}(\mathbf{B}), I_{2}=\left[(\operatorname{tr} \mathbf{B})^{2}-\operatorname{tr} \mathbf{B}^{2}\right] / 2, I_{3}=\operatorname{det}(\mathbf{B})=J^{2}
$$

For a nearly incompressible Gent model the strain energy is given by:

$$
W=-\frac{\mu}{2} J_{m} \ln \left(1-\frac{I_{1}-3}{J_{m}}\right)-\mu \ln J+\left(\frac{K}{2}-\frac{\mu}{J_{m}}\right)(J-1)^{2}
$$

Where $\mu$ and $K$ are the initial shear and bulk moduli, respectively, and $J_{m}$ is a constant related to the strain saturation of the material. The Cauchy stress, a measure of the force acting on an element of area in the deformed material, is given by

$$
\boldsymbol{\sigma}=\frac{2}{J} \frac{\partial W}{\partial I_{1}} \mathbf{B}+\frac{\partial W}{\partial J} \mathbf{I}
$$

yielding the expression

$$
\boldsymbol{\sigma}=\frac{\mu J_{m}}{J\left(J_{m}-I_{1}+3\right)} \mathbf{B}-\frac{\mu}{J} \mathbf{I}+\left(K-\frac{2 \mu}{J_{m}}\right)(J-1) \mathbf{I}
$$

with the corresponding nominal stress $\mathbf{s}=J \boldsymbol{\sigma} \cdot \mathbf{F}^{-\mathbf{T}}$, a measure of the force acting on an element of area in the undeformed, original configuration, being 


$$
\mathbf{s}=\frac{\mu J_{m}}{J_{m}-I_{1}+3} \mathbf{F}-\mu \mathbf{F}^{-\mathbf{T}}+\left(K-\frac{2 \mu}{J_{m}}\right) J(J-1) \mathbf{F}^{-\mathbf{T}}
$$

For the analysis, the parameters $\mu, J_{m}, K$ were obtained by fitting to experimental load-displacement curves measured in tension (see figure 6), yielding values $\mu=0.06 \mathrm{MPa}, K$ $=24 \mathrm{MPa}$ and $J_{m}=28.2$. To account for the pre-stretch, $\chi$, the deformation gradient $\mathbf{F}$ is decomposed into a load-induced gradient, $\mathbf{F}^{\mathbf{L}}$, and a gradient produced by pre-stretching, $\mathbf{F}^{\mathbf{S}}$, following the multiplicative decomposition method originally introduced by Kroner-Lee ${ }^{20,21}$,

$$
\mathbf{F}=\mathbf{F}^{\mathbf{L}} \mathbf{F}^{\mathbf{s}}, \quad \text { with } \quad \mathbf{F}^{\mathbf{s}}=\operatorname{diag}(\chi+1,1 / \sqrt{\chi+1}, 1 / \sqrt{\chi+1})
$$

This material behavior described by equations (3) to (7) was implemented into the commercial software ABAQUS/Standard and ABAQUS/Explicit through user defined material subroutines and used to investigate the full deformation response of the bi-strips.

\section{Eigenvalue Analysis.}

The stability of the bi-strips was first examined using standard eigenvalue analyses. A linear perturbation procedure that fully accounts for the pre-loading was used and implemented within the commercial finite element code ABAQUS/Standard using the *BUCKLE module. Models meshed with 3D quadratic reduced integrations elements (ABAQUS element type C3D20R) were built and tested and the accuracy of the mesh was ascertained through a mesh refinement study.

In the simulations, to mimic the experiments, one end of the bi-strip was rigidly displaced towards the fixed end without allowing any rotation until at a displacement $\left(\Delta L_{\text {crit }}\right)$, relative to the initial, pre-strained length, the bifurcation point (indicated by a zero eigenvalue for the stiffness matrix of the bi-strip) was found. By performing this for a series of pre-strains, the critical strain $\Delta L_{\text {crit }} / L$ can be obtained as shown in Figure 7.

The calculations indicate that multiple instability modes consisting of alternating regions of opposite chirality do form and the critical strains at which these occur are very closely spaced as shown in figure 7 . The first eigenmode is characterized by two regions of opposite chiralities 
with one perversion, the second mode consists of a sequence of three regions of opposite chiralities and two perversions, the third mode consists of four regions of opposite chirality with three perversions and so on with the number of perversions monotonically increasing linearly with the eigenmode number. The appearance of closely spaced modes is in marked contrast to buckling in many other systems, including the classical Euler buckling of columns in compression for instance, where the eigenvalues are well separated and the one with the lowest eigenvalue invariably grows. Furthermore, the critical strains are very small $(\sim 0.1)$ indicating that the buckling instability with twist occurs almost as soon as the ends are released. This, too, is consistent with figure 1. Moreover, according to Koiter's theory we expect the instability to occur at a lower load because of imperfections in the samples. As in the analysis of other elastic buckling situations, the mode selection and hence, in this case the number of perversions, need to be determined by a post-buckling analysis ${ }^{22}$.

Finally, consistent with the experimental observations, the response of the bi-strips is found not to be affected by the choice of whether the boundary conditions are both fixed or one is fixed and the other free to rotate, confirming that the formation of the hemi-helix is a robust and deterministic process.

\section{Post-buckling analysis.}

To simulate the large displacement behavior of the bi-strips after the onset of buckling, we used numerical analyses. Static finite element analyses were effective in determining the number $N$ of perversions in the system shortly after buckling but they were not suitable to simulate the complete release process. In part this was because of the large geometric changes and the material non-linearity. To capture the response behavior over the entire unloading path, dynamic explicit simulations were performed within the ABAQUS/Explicit software. For this set of simulations, models with 3D linear reduced integration elements (ABAQUS element type C3D8R) are used and quasi-static conditions were ensured by monitoring the kinetic energy and introducing a small damping factor. In all the simulations, the accuracy of each mesh was ascertained through a mesh refinement study.

To illustrate that the dynamic explicit method captures the experimental observations,

the release of the bi-strip in figure 1 with a pre-strain of $\chi=1.5$ and initial length of $50 \mathrm{~cm}$ with 
both ends unable to rotate was simulated. Images from the simulation are reproduced in figure 8 (a) to illustrate the development of the hemi-helix on release to the lengths indicated in the figure. Random imperfections were included in the finite element model by successively running the model after randomly perturbing the positions of the individual elements. It was found that irrespective of the type of random imperfection introduced, the eleventh eigenmode grew, consistent with the observations in figure 1. A higher magnification snap-shot taken from another simulation, in this case of a $25 \mathrm{~cm}$ long bi-strip and pre-strain of $\chi=0.5$, is shown in figure $8(\mathrm{~b})$ as a direct comparison with the micro-CT image in figure 3 . The distinct regions where twisting and bending deformation predominate are faithfully reproduced and the perversions can also be distinguished in both images. Post-buckling computations for bistrips of different cross-sectional geometries and having different pre-strains are compared with experimental data in figure 5. Excellent agreement between the simulations and experiment is seen over the range of pre-strain values and cross-sectional geometries investigated. Furthermore, the numerical analysis also correctly captures the dependence of $N$ on the pre-strain and strip dimensions. We believe that the slight discrepancies can be explained by differences in the constitutive behavior of the elastomer and the idealization of the end effects compared with the finite extent of the gripped ends in the experiments.

\section{Elastic Energies}

The numerical simulations using the strain energy formulation of equation 1 enables the energies of the hemi-helix to be directly compared to those of a simple helix and address the question of whether or not the hemi-helix is a configuration trapped in a higher energy state as a result of the onset of the buckling instability associated with the formation of the hemi-helix and perversions. Since there are complex, nonlinear coupling between the twist, bending and stretching contributions to the overall strain energy of the hemi-helix, and the observations suggest that there is a repulsive interaction between perversions, we simulated the energy of the simplest hemi-helix containing just one perversion. Using the data in figure $5(\mathrm{a})$, a $10 \mathrm{~cm}$ long bi-strip of equal $3 \times 3 \mathrm{~mm}$ cross-section strips and a pre-strain $\chi=0.5$ was selected. The elastic energy was then computed as the two, fixed ends were slowly displaced towards one another and the instantaneous shape monitored. The dynamics simulations were performed sufficiently slowly to ensure that the kinetic energy was zero throughout. As shown in the 
results in figure 9, the elastic energy decreases nonlinearly with displacement and at a critical displacement the bi-strip no longer remains flat and bifurcation occurs, at point $A$, to form a hemi-helix with a single perversion. Thereafter, the elastic energy decreases further, again nonlinearly, with continued end displacement and the amplitude of the hemi-helix increases. At an arbitrary displacement, at $30 \mathrm{~mm}$ in figure 9 , point $C$, one end is rotated without any end displacement to remove the perversion converting the hemi-helix to a regular helix. There is initially a slight increase in energy, $(0.235 \mathrm{~mJ})$, from $C$ to $C^{\prime}$, corresponding to having to overcome an energy barrier in rotating the end, and then the elastic energy drops to point $D$. In figure 9 this drop is $0.735 \mathrm{~mJ}$. The energy change and energy barrier is shown in the insert of the figure. The shapes just before and after the end rotation, $C$ and $D$ respectively, are also shown in figure 9 for comparison. As the displacement of two ends towards one another is continued, the energy decreases further as shown by the segment $D E$ in the figure. If the displacement direction is then reversed so that strained helix at point $E$ is now elongated, the energy path is reproduced until the $30 \mathrm{~mm}$ displacement is reached and then the energy follows the lower path shown in figure 9. Not only is the path lower in energy but it has a shallower dependence on displacement. When the simulation was re-run from point $C$ but without rotating one of the ends, the strain energy path continued smoothly along the original path to the point $F$.

The energy simulations confirm that the hemi-helix morphology indeed has a higher elastic energy than that of the simple helix. Furthermore, the energy of a perversion depends on the stretching strain, presumably through a twist-elongation coupling. Thus it can be expected that the energy drop in figure 9 at a displacement of $30 \mathrm{~mm}$ is significantly smaller than the energy difference between the hemi-helix and the helix at smaller displacements. This is suggested by the steeper slope of the hemi-helix energy curve compared with that of the helix extension about point $D$. 


\section{DISASSION}

As mentioned in the Introduction, the three-dimensional hemi-helical configuration formed by the relaxation of a pre-stretched and planar bi-strip is a new geometry. Unlike previous reports of the buckling of a slightly bent uniform rod ${ }^{11}$ where a single perversion forms, the hemi-helix consists of a periodic arrangement of multiple perversions separating regions of opposite chiralities. Experiments over a range of pre-strains and cross-sectional dimensions indicate that the number of perversions and their separation depend on these parameters, findings substantiated by finite element simulations of both the buckling and postbuckling analysis. The simulations provide the important insight that the hemi-helix forms as a result of the early onset of a buckling instability associated with out-of-plane twisting of the bistrip rather than a bending instability that leads to the formation of a normal helix.

The simulations also reveal that, in contrast to other buckling phenomena, such as standard Euler buckling of a column, the possible eigen-modes are closely spaced in wavelength indicating that there are a variety of possible closely-bunched bifurcations. Because the deformation of the pre-strained bi-strip is so complex, incorporating extensile and bending as well as twisting strains, simple closed-form analysis of the mode selection and deformation paths has not been feasible. This is unlike column buckling where only bending and extensile strains are involved, and where analytical solutions are possible. Nevertheless, the fact that the simulations of the experimental observation in figure 1 predict that the eleventh eigenmode grows, consistent with what is observed, suggest that the simulation correctly incorporates the full deformation behavior. Furthermore, the consistency between the perversion separation and the pre-strain simulated and observed provides further support that the formation of the hemi-helices is fully deterministic as suggested by the experimental findings. The physical rationale for the observed scaling of the perversion wavelength and the pre-strain and crosssectional geometry remains to be understood but provides further evidence for the hemi-helix formation being deterministic and robust, at least for the slender geometry of the bi-strips studied.

The simulations also resolved a question not amenable to experiment, namely whether hemi-helices form in linear elastic materials as distinct from highly non-linear materials. To date we have not found any linear elastic material that can sustain the large pre-strains 
necessary to form hemi-helices without breaking and hence could not resolve the question experimentally. However, the simulations indicate that the occurrence of the hemi-helix does not depend on the constitutive behavior of the material. Indeed, post-buckling modeling of bistrips made of linear elastic materials also reveals that hemi-helices form upon release. More specifically, when we simulated a linear elastic bi-strip having dimensions $w=h=3 \mathrm{~cm}$ in which both strips had a shear modulus and bulk modulus of $0.06 \mathrm{MPa}$ and $24 \mathrm{MPa}$, respectively, and a pre-strain $\chi=0.5$, hemi-helices with an initial wavelength of $12.23 \mathrm{~cm}$ formed when the ends were released. The initial buckling wavelength of the hemi-helices formed is only slightly different than when the constitutive behavior of figure 6 is used $(12.045 \mathrm{~cm})$. Further simulations are clearly necessary to explore the dependence on elastic modulus, pre-strain and geometric parameters and are underway but this simulation demonstrates that the formation of the hemi-helix is not dependent on the non-linearity of the elastic material.

Although the hemi-helix configuration is stable and is always observed in our experiments, this does not mean that it has the lowest energy for all possible configurations. For instance, as illustrated by our simulations, a different loading path can be constructed to change the bi-strip hemi-helix into a helix: after the hemi-helix is formed and the ends moved towards one another, one end can be twisted. In rotating one end, the elastic energy is decreased, which indicates that the helix has a lower energy than the hemi-helix at the same distance between the ends, but an energy barrier must first be overcome, as is illustrated in the insert of figure 9. This can also be understood qualitatively: if one starts with either a right or left handed helix and with one end fixed, the other end is wound in the counter-direction to create a hemi-helix, work must be done in twisting the helix. Furthermore, the larger the number of perversions that are introduced, the more work that must be done. The dynamic simulations show that as the hemi-helices are turned into helices by a snap-through process there is transient wave propagation, as observed in the experiments. More detailed simulations are necessary to determine whether the twisting bifurcation resulting in the formation of the hemi-helix occurs because there is an insurmountable energy barrier to be overcome in adopting the helical shape.

In future work, the range of cross-sectional geometries and pre-strains over which a hemi-helix forms needs to be clarified. Since the hemi-helix forms as a result of an out-of-plane 
twisting deformation, it can be anticipated that as the height-to-width aspect ratio of the bistrip is increased, twisting will not be the energetically preferred initial instability. Thus, one can anticipate that hemi-helices will only form for a certain range of geometric ratios between height and width. Outside of this range, normal helical morphological shapes can be expected. This expectation was confirmed by performing a numerical simulation for a larger aspect strip, $h / w=20 / 3$ and the same pre-strain as in figure 1 . A series of images from the simulation sequence is shown in figure 10 which clearly indicates that a simple helix does form. Also, in those cases where the pre-straining does not give rise to appreciable twist, hemi-helices will not be expected to form. This is perhaps the reason that the three-dimensional shapes by Chen et al. ${ }^{8}$ were all regular helices without the formation of any hemi-helices.

The formation of multiple perversions in a single strip provides an opportunity to study the essential physics of perversions as a "defect" or "singularity" in materials. (We use the term "defect" in the same sense that magnetic and ferroelectric domain walls, for instance, are defects that form in materials in response to the imposed boundary conditions and increase the total energy of the material. By changing the boundary conditions, the "defects" can move as we have demonstrated by slowly rotating one end of a hemi-helix much as domain walls move in response to changing the external field, for instance). The observations reported in this work indicate that topologically they separate regions of opposite chirality, that they separate regions of predominately twist and bending deformation, and that they repel one another. They also have an excess elastic energy associated with them and presumably also a rotational strain gradient that causes them to repel.

Finally, although formally the hemi-helix shape can be obtained by winding a simple helix in the opposite direction to its existing chirality, the important result of this paper is that the shape can be produced spontaneously by constructing a long, pre-strained bi-strip and releasing it. Irrespective of the end conditions during the release, the shape is reproducible and the number of perversions and their wavelength are fully determined by the cross-section geometry and the value of the pre-strain. These features enable the hemi-helix, a three-dimensional shape, to be designed and produced from two-dimensional strips for practical applications. These might be elastic springs with unusual non-linear behavior for MEMS applications or light filters if one of the strips is made of a chiral polymer, for instance. 


\section{CONOLUSIONS}

Upon release, a simple stretched bi-strip of elastomers, consisting of a long pre-strained strip attached side-by-side to an unstrained strip, undergoes a complex buckling instability to form a hitherto unreported three-dimensional shape. We term this new shape a hemi-helix. It consists of multiple, alternating helical sections of opposite chiralities. Separating the regions of opposite chiralities are perversions. The geometry of the hemi-helix is determined by the prestrain, the cross-section and the constitutive deformation behavior of the elastomer. Also, unlike the shapes produced by swelling of gels produced by changes in $\mathrm{pH}^{23}$ or by differential

cross-linking ${ }^{24}$, the pre-straining operation is characterized by a well defined strain. Numerical simulations indicate that the hemi-helix forms preferentially because the initial instability involves twisting as well as bending and extension. The simulations also show that the hemihelix has a higher energy than the corresponding helix formed when the ends of the hemi-helix are rotated to remove the perversions. Furthermore, the formation of the hemi-helix is not a result of the non-linear elastic behavior of the elastomers since simulations show that they can also form with linearly elastic materials. It does, however, require that the materials have the capability of large strain, elastic behavior and so seems to form only in soft materials with large extensibility to failure, such as elastomers.

\section{EXPERIMENTALPROCEDURES}

The strips of material used in the experiments were elastomers cut from platinum cured silicone rubber sheets formed from a two-part commercial product (Dragon Skin 10 Slow, Reynolds Advanced Materials). The elastomer was cast as sheets between two large parallel acrylic sheets $(20 \times 60 \mathrm{~cm})$ held $3 \mathrm{~mm}$ apart and cured for 7 hours at room temperature. Coloring agents (Silicone pigment, Reynolds Advanced Materials) were added during mixing of the elastomer before casting. The elastomer does not stick to the acrylic sheet and after curing was simply peeled away. The strips were flat and undistorted after peeling away the sheets indicating that they were free of any residual stress. The strips were then scored into strips with a laser beam and cut using a blade. The glue used to bond the strips together was a silicone rubber product (Sil-Poxy) also purchased from Reynolds Advanced Materials. 


\section{APPENDIX ONE}

A line that forms a helix with a radius $R$ and repeats by a distance $2 \pi b$ per turn, can be described in Cartesian coordinates by the parametric equation:

$$
p(t)=(x(t), y(t), z(t))=\{R \sin (t), R \cos (t), b t\}
$$

where $p(t)$ is a position vector of a point located a distance $t$ along the helical line. A line having the ideal hemi-helix geometry with periodic perversions can similarly be expressed as:

$$
p(t)=\{R \cos (\pi \sin (t)), R \sin (\pi \sin (t)), b t\}
$$

This function exhibits periodic reversals in chirality with two perversions repeated every $2 \pi b$ along the z-direction. Topologically, the hemi-helix has zero net twist and a writhe number of zero. 


\section{APPENDIX TWO}

\section{ANALYSIS OF THE BENDING RADIUS}

We present an analytical expression for the helical radius of curvature on the tangential plane for a fully released bi-strip. Under the assumption that the configuration is dominated by bending, we estimate the radius of curvatures from a simple bending analysis. Thus, a uniaxial state of stress is assumed where only the normal stress along the longitudinal direction is nonvanishing,

$$
\boldsymbol{\sigma}=\operatorname{diag}\left(\sigma_{11}, 0,0\right)
$$

To derive explicit formula we assume a fully incompressible elastomer (i.e. det $F=1$ ) so that the state of deformation in each strip is fully characterized by

$$
\mathbf{F}=\operatorname{diag}(\lambda, 1 / \sqrt{\lambda}, 1 / \sqrt{\lambda})
$$

For the sake of simplicity we focus on a portion $\Delta L$ of the bi-strip characterized by a 2D fanshape in the release state with the major radius contained in the plane. (Figure A1). $R$ is the radius of curvature of the outer boundary which has undergone a longitudinal stretch, $\tilde{\lambda}$ (defined as current length of a line segment divided by its original length).

According to elastic beam theory and the assumption of pure bending, the displacement on the cross-section is linearly dependent on the width coordinates $\xi$. The longitudinal stretch at an arbitrary point on the cross-section of the two strips is given by

$$
\lambda_{a}=\frac{R-\xi}{R} \tilde{\lambda}, \lambda_{b}=\frac{R-\xi}{R} \tilde{\lambda}(1+\chi)
$$

The subscripts $a$ and $b$ denote quantities related to the non-prestretched and prestretched strips.

For an incompressible Gent material, the strain energy [eqn. (3) in the text] simplifies to 


$$
W=-\frac{\mu J_{m}}{2} \ln \left(1-\frac{I_{1}-3}{J_{m}}\right), \text { where } I_{1}=\operatorname{tr}(\mathbf{B})=\operatorname{tr}\left(\mathbf{F} \mathbf{F}^{\mathrm{T}}\right)
$$

and the Cauchy stress is then

$$
\boldsymbol{\sigma}=-p \mathbf{I}+2 \frac{\partial W}{\partial I_{1}} \mathbf{B}=-p \mathbf{I}+\frac{\mu J_{m}}{J_{m}-I_{1}+3} \mathbf{B}
$$

where $p$ is the hydrostatic pressure whose value is determined by the boundary conditions. In each strip the pressure can be solved from the condition $\sigma_{22}=\sigma_{33}=0$ yielding

$$
p=\frac{\mu J_{m}}{\lambda\left(J_{m}-I_{1}+3\right)}, \text { with } \quad I_{1}=\lambda^{2}+\frac{2}{\lambda}, \text { so that } \sigma_{11}=\left(\lambda^{2}-\frac{1}{\lambda}\right)\left(\frac{\mu J_{m}}{J_{m}-I_{1}+3}\right)
$$

Finally, mechanical equilibrium requires that the net forces and moments are zero, yielding

$$
\begin{array}{r}
\int_{0}^{w_{a}} \sigma_{a} h_{a} d \xi+\int_{w_{a}}^{w_{a}+w_{b}} \sigma_{b} h_{b} d \xi=0 \\
\int_{0}^{w_{a}} \sigma_{a} h_{a} \xi d \xi+\int_{w_{a}}^{w_{a}+w_{b}} \sigma_{b} h_{b} \xi d \xi=0
\end{array}
$$

where $\sigma_{a}$ and $\sigma_{b}$ and the current heights $h_{a}$ and $h_{b}$ in the cross-section are functions of $\xi$ with

$$
h_{a}=\frac{1}{\sqrt{\lambda_{a}}} h, h_{b}=\frac{1}{\sqrt{\lambda_{b}}} h
$$

Moreover, note that the width of each strip entering the evaluation of the integrals is obtained using the longitudinal stretch at the mid-plane in the current state,

$$
w_{a}=\frac{w}{\sqrt{\lambda_{a}\left(\xi=\frac{w_{a}}{2}\right)}}, w_{b}=\frac{w}{\sqrt{\lambda_{b}\left(\xi=w_{a}+\frac{w_{b}}{2}\right)}}
$$


Equations (A6) to (A9) cannot be solved analytically, so the "trust-region-dogleg" algorithm within Matlab software was used to solve numerically for the outer layer radius $\mathrm{R}$ and the stretch $\tilde{\lambda}$ in terms of the pre-strain, $\chi$. The results are reported in Figure 4 in the text along with the experimental data. Note that the main radius of curvature is only influenced by material and geometry parameters, and so the predicted outer radius curvature is the same for both the hemi-helix and simple helix.

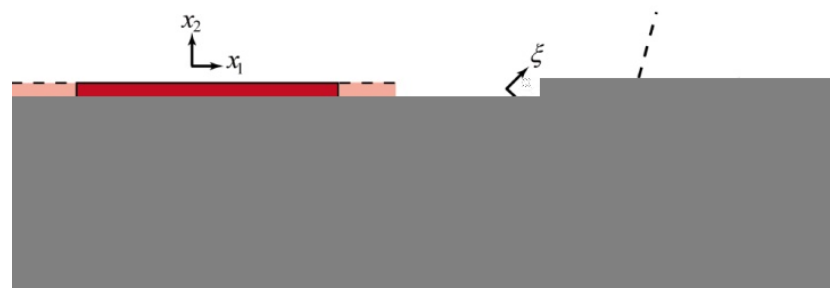

Figure A1. Schematic figures for a small portion of strips in the initial and fully released state. The straight portion (with a length $\Delta L$ ) deforms into a fan-shape segment, leading to a curved outer layer with major radius of curvature $R$ and length $\tilde{\lambda} \Delta L$. Subscripts $\mathrm{a}$ and $\mathrm{b}$ are used to describe the non-prestretched and prestretched strips, respectively.

\section{ACKNOWLEDGEMENTS}

This work was supported by the DARPA Bio-Inspired Photonics Program, contract W911NF-101-0113. The authors are grateful to discussions with Lev Trushinovsky, David Cannell, Basile Audoly and Tiefeng $\mathrm{Li}$ and to Tom Hughes for suggestions on efficient procedures for modeling post-buckling dynamics. Benedikt Kroll's research was supported under the auspices of the National Science Foundation's Harvard MRSEC program grant DMR-0820484 and its REU program.

\section{CORRESPONDING AUTHOR}

Correspondence and requests for materials should be addressed to clarke@seas.harvard.edu. 


\section{FIGURECAPTIONS}

Figure 1. Sequence of images (top to bottom) recorded as a bi-elastomer strip, of initial length $50 \mathrm{~cm}$ and pre-strain $\chi=1.5$, is released by moving the ends of the bi-strip together. (The lengths are indicated on the right hand side of the figure and the grips holding the ends are not shown). Even at the earliest stages of releasing the ends, the perversions (arrowed and eleven in number) begin to form. After release, the coiled bi-elastomer strip adopts the shape shown in (b). For clarity, the coiled strip is laid out as a straight strip. The alternative configuration (c) of the same strip and shown at the same magnification, consisting of a tightly wound coil with the inner portion being red and the outer portion being blue-green, is adopted if the ends are twisted by hand after releasing the ends. Note, the two strips are made of identical material but dyed red and green to distinguish them and the magnifications of the individual images are different in figure (a).

Figure 2. The pre-straining operation used to form the strained bi-strip. (a) The initial geometry of the elastomeric strips before pre-straining. (b) The red strip is stretched by a force until it has the same length as the blue-green strip. The two strips are then glued together. The prestrain is defined as: $\chi=\left(L-L^{\prime}\right) / L^{\prime}$

Figure 3. Detail of the hemi-helix imaged by micro X-ray computerized tomography. The spatial separation into regions of predominately twisting and bending is apparent. The approximate positions of the perversions are indicated by the dashed vertical lines and the regions of pronounced twisting are highlighted by the dashed circles. As can be seen the perversions are located in the regions of pronounced bending.

Figure 4. The radius of curvature as a function of pre-strain for both hemi-helices and helices after stretching and releasing them. Red circles and black squares are the experimental results for hemi-helices and helices, respectively. The blue line is the analytical prediction from Appendix Two. The radius of curvature decreases with pre-strain since larger pre-strains produce larger differential stresses and thus the bi-strip system has a smaller radius of curvature. 
Figure 5 (a). The initial hemi-helical wavelength as a function of the pre-stretch ratio for red and blue-green strips each of equal width and height $(\mathrm{w}=\mathrm{h}=3 \mathrm{~mm})$ and initial length of $50 \mathrm{~cm}$. The line through the data corresponds to a power law dependence on $\chi$ with an exponent of $1 / 3$. The results of the numerical solution simulation using a hyper-elastic Gent model for the constitutive behavior are indicated. Figure $\mathbf{5}$ (b). Variation in the initial hemi-helical wavelength as a function of the width and height of the individual strips for fixed pre-stain of 0.5 . Initial bistrip length of $50 \mathrm{~cm}$. The simulation results are included for comparison. Figure 5 (c). Experimentally determined scaling between the initial wavelength and the parameter $\mathrm{w}^{2 / 5} \mathrm{~h}^{3 / 5}(\chi)^{-1 / 3}$, again with the simulation results for comparison. The error bars in the experimental data are approximately the same size as the symbols.

Figure 6. The uniaxial stress-stretch response of the elastomer material used to produce the bistrips. The red circles correspond to the experimental data, while the continuous curve corresponds to the best fit to the hyper-elastic Gent model. Here, the stretch $\lambda$ is defined as current length dividing by the original length. The nominal stress, $s$, is obtained as the force divided by the original area.

Figure 7. The calculated critical applied strain at which buckling occurs for different eigenmodes. Initial strip length $50 \mathrm{~cm}$ and $\mathrm{w}=\mathrm{h}=3 \mathrm{~mm}$. Note that the critical strains for the different eigenmodes are very close to one another and that the critical strain increases only slightly with the pre-stretch ratio, $\chi$.

Figure 8. (a) A series of images from the simulation of the release of a $50 \mathrm{~cm}$ long bi-strip prestrained by $\chi=1.5$ simulating the hemi-helix in figure 1 . (b) A snap-shot taken from a simulation sequence of the release of a $25 \mathrm{~cm}$ bi-strip, pre-strained by $\chi=0.5$. The variation in von Mises stresses are shown by the color scale. As in the micro-computerized tomography image of figure 3, alternating regions of predominately bending and twisting form along the length of the hemi-helix as well as the perversions. (The von Mises stress is a scalar given by the equation:

$$
2 \sigma_{V}^{2}=\left\lfloor\left(\sigma_{11}-\sigma_{22}\right)^{2}+\left(\sigma_{22}-\sigma_{33}\right)^{2}+\left(\sigma_{11}-\sigma_{33}\right)^{2}+6\left(\sigma_{23}^{2}+\sigma_{31}^{2}+\sigma_{12}^{2}\right)\right\rfloor
$$


Figure 9. Elastic strain energy of a $10 \mathrm{~cm}$ long bi-strip as its two ends are gradually displaced towards one another. At a displacement of $10 \mathrm{~mm}$, point $A$, the straight bi-strip becomes unstable to twisting and a hemi-helix with a single perversion forms. With further displacement, the elastic energy continues to decrease. At an arbitrary displacement of $30 \mathrm{~mm}$, point $C$, the displacement is paused and one end is rotated to remove the perversion and form a helix. The elastic energy first increases to $C^{\prime}$ and then drops to point $D$ as shown in the insert, indicating that there is an excess energy associated with the perversion. With continued end displacement, the energy decreases continuously along path $D E$ (in blue). When the direction of displacement is reversed, the path $E D$ is retraced followed by the lower path shown in red. If the end is not rotated, the energy path continues from $C$ to $E$ (green). Pre-strain, $\chi=0.5$ and cross-section $\mathrm{w}=\mathrm{h}=3 \mathrm{~mm}$. For clarity only one third of the computed data points are shown.

Figure 10. Three snap-shots from the simulation sequence of the release of a wide bi-strip, $h / w=20 / 3$, indicating the formation of a helix rather than a hemi-helix. Length $50 \mathrm{~cm}$ and pre-strain $\chi=0.5$.

Figure A1. Schematic figures for a small portion of strips in the initial and fully released state. The straight portion (with a length $\Delta L$ ) deforms into a fan-shape segment, leading to a curved outer layer with major radius of curvature $R$ and length $\tilde{\lambda} \Delta L$. Subscripts a and $\mathrm{b}$ are used to describe the non-prestretched and prestretched strips, respectively. 


\section{REFERENCES}

1. D. A. Thompson, On Growth and Form, Cambridge University Press, 1961.

2. P. Ball, The Self-Made Tapestry: Pattern Formation In Nature, Oxford University Press, 1999.

3. T. A. Witten and L. M. Sander, Physical Review Letters, 1981, 47, 1400.

4. S. Wolfram, A New Kind of Science, Wolfram Media, Inc, 2002.

5. E. Sharon and E. Efrati, Soft Matter, 2010, 6, 5693-5704.

6. T. Savin, N. A. Kurpios, A. E. Shyer, P. Florescu, H. Liang, L. Mahadevan and C. J. Tabin, Nature, 2011, 476, 57.

7. S. Armon, E. Efrati, R. Kupferman and E. Sharon, Science, 2011, 333, 1726.

8. Z. Chen, C. Majidi, D. J. Srolovitz and M. Haataja, Applied Physics Letters, 2011, 98, 011906.

9. Y. Sawa, F. Ye, K. Urayama, T. Takigawa, V. Gimenz-Pinto, R. L. B. Selinger and J. V. Selinger, Proceedings of the National Academy of Sciences, 2011, 108, 6364-6368.

10. R. Oda, I. Huc, M. Schmutz, S. J. Candau and F. C. MacKintosh, Nature, 1999, 399, 566569.

11. T. McMillen and A. Goriely, Journal of Nonlinear Science, 2002, 12, 241-281.

12. P. Pieranski, J. Baranska and A. Skjeltorp, European Journal of Physics, 2004, 25, 613621.

13. R. Goldstein, A. Goriely, G. Hubber and C. Wolgemuth, Physical Review Letters, 2000, 84, 1631-1634.

14. N. Ousaka and Y. Inai, Journal of the American Chemical Society, 2006, 128, 1473614737.

15. L. D. Landau and E. M. Lifshitz, Statistical Physics. Part 1., Pergamon Press, 1980.

16. P. M. Chaikin and T. C. Lubensky, Principles of Condensed Matter Physics, Cambridge University Press, Cambridge, 1995.

17. M. Hetenyi, Beams on Elastic Foundations, Waverly Press, Baltimore, 1946.

18. R. W. Odgen, Non-Linear Elastic Deformation, Dover Editions.

19. A. N. Gent, Rubber Chemistry and Technology, 1996, 69, 59.

20. E. Kroner, Archive for Rational Mechanics and Analysis, 1960, 4, 273.

21. E. H. Lee, Journal of Applied Mechanics, 1969, 36, 1.

22. J. W. Hutchinson and W. T. Koiter, Applied Mechanics Reviews, 1970, 23, 1353-1365.

23. E. Sharon, M. Marder and H. L. Swinney, American Scientist, 2004, 92, 254.

24. J. Kim, J. A. Hanna, C. D. Santangelo and R. C. Hayward, 2011. 


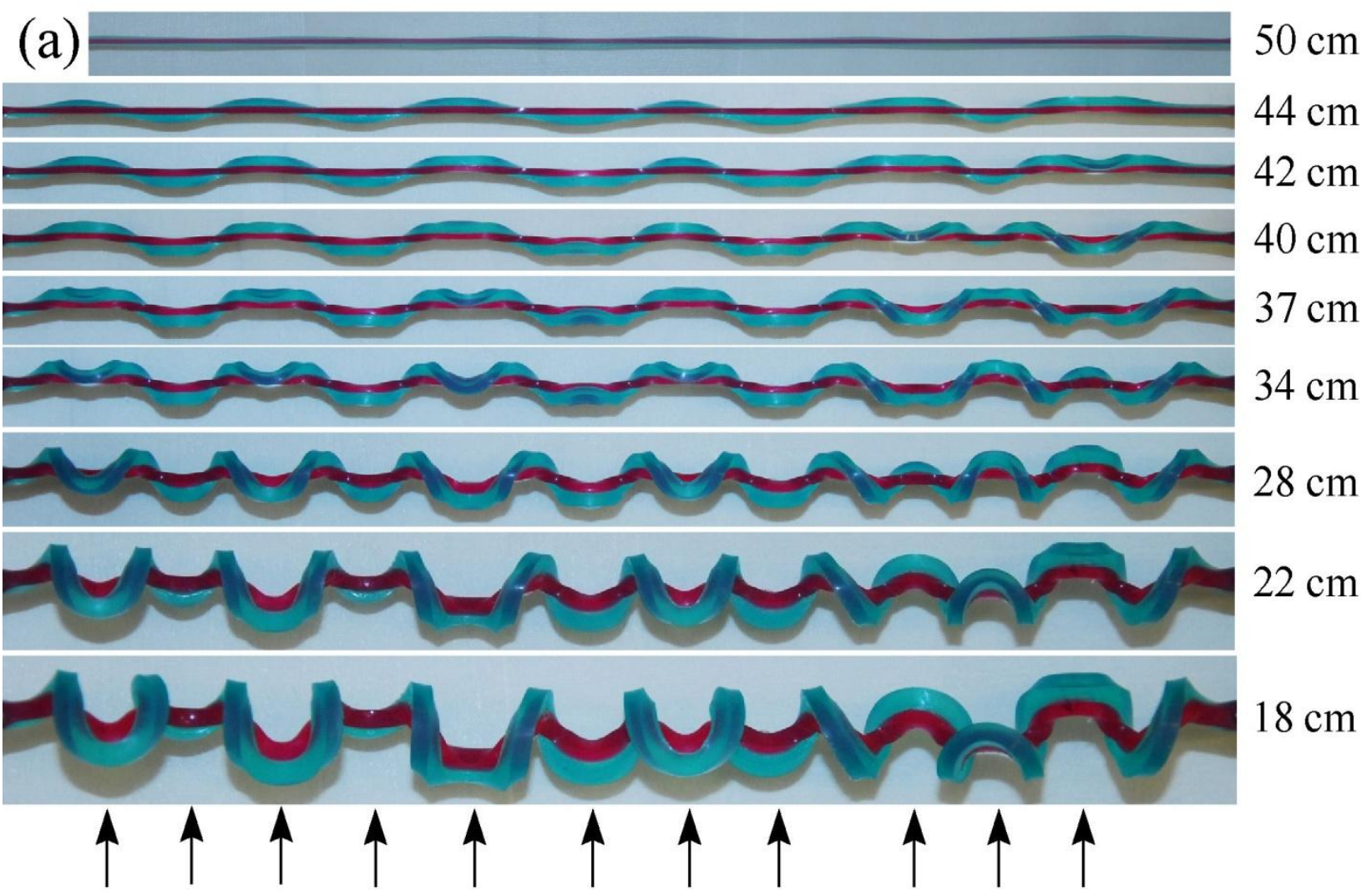

(b)

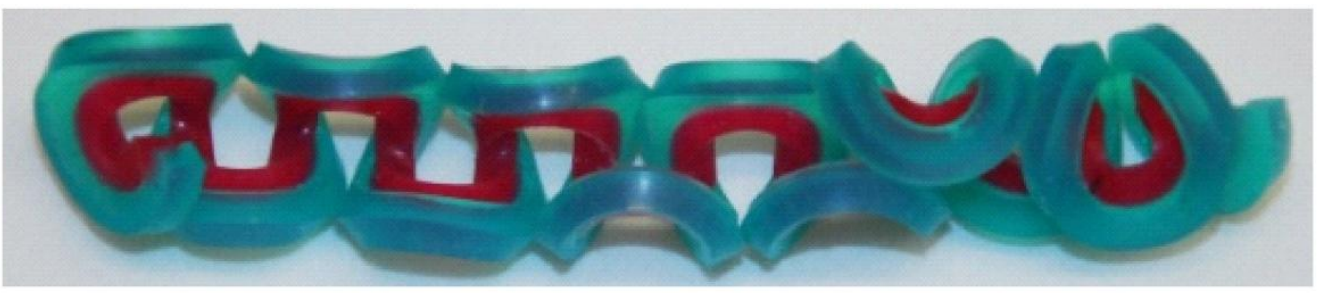

(c)

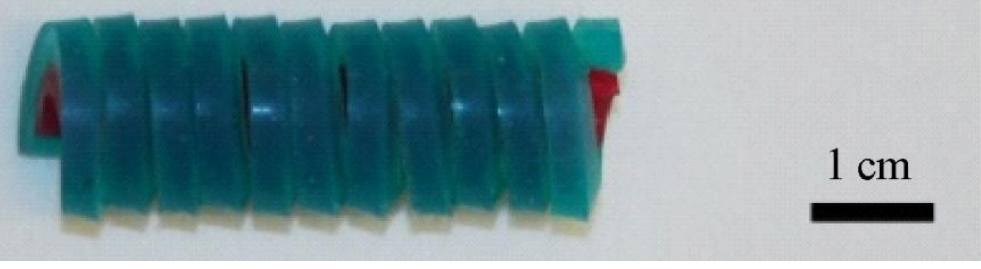

Figure 1. Huang et al. 

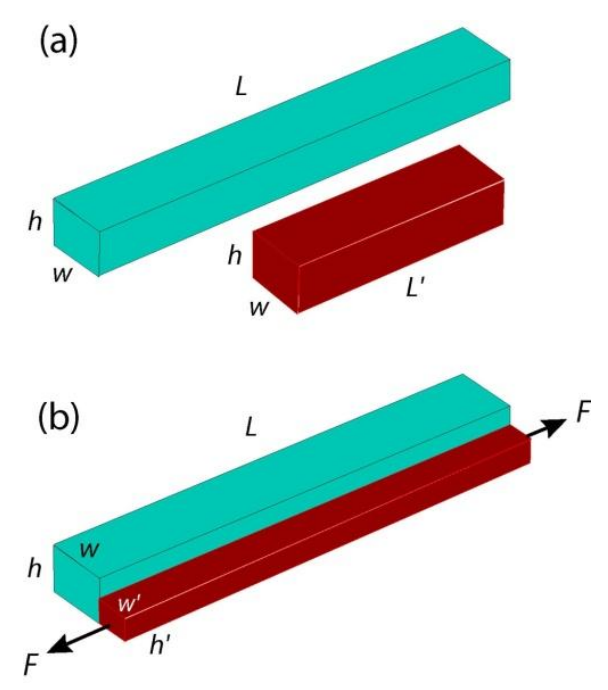

Figure 2. Huang et al.

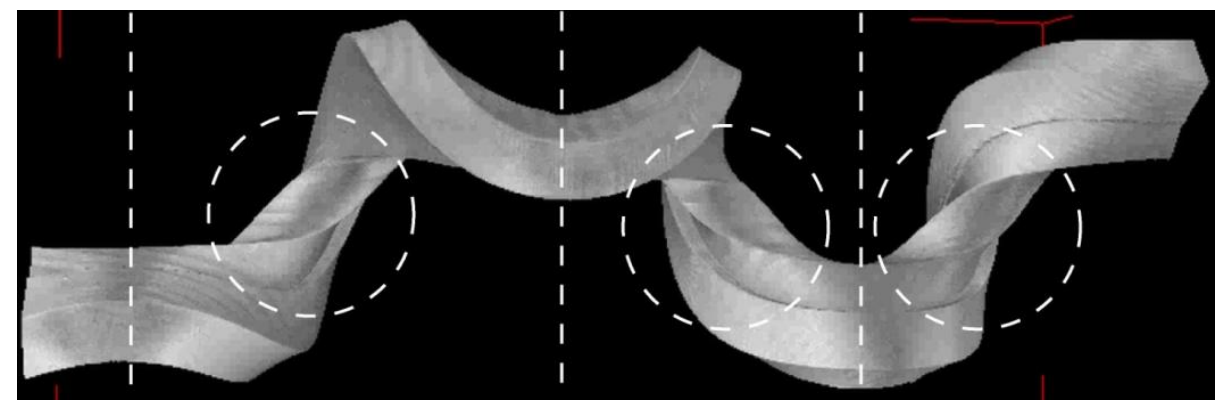

Figure 3. Huang et al. 


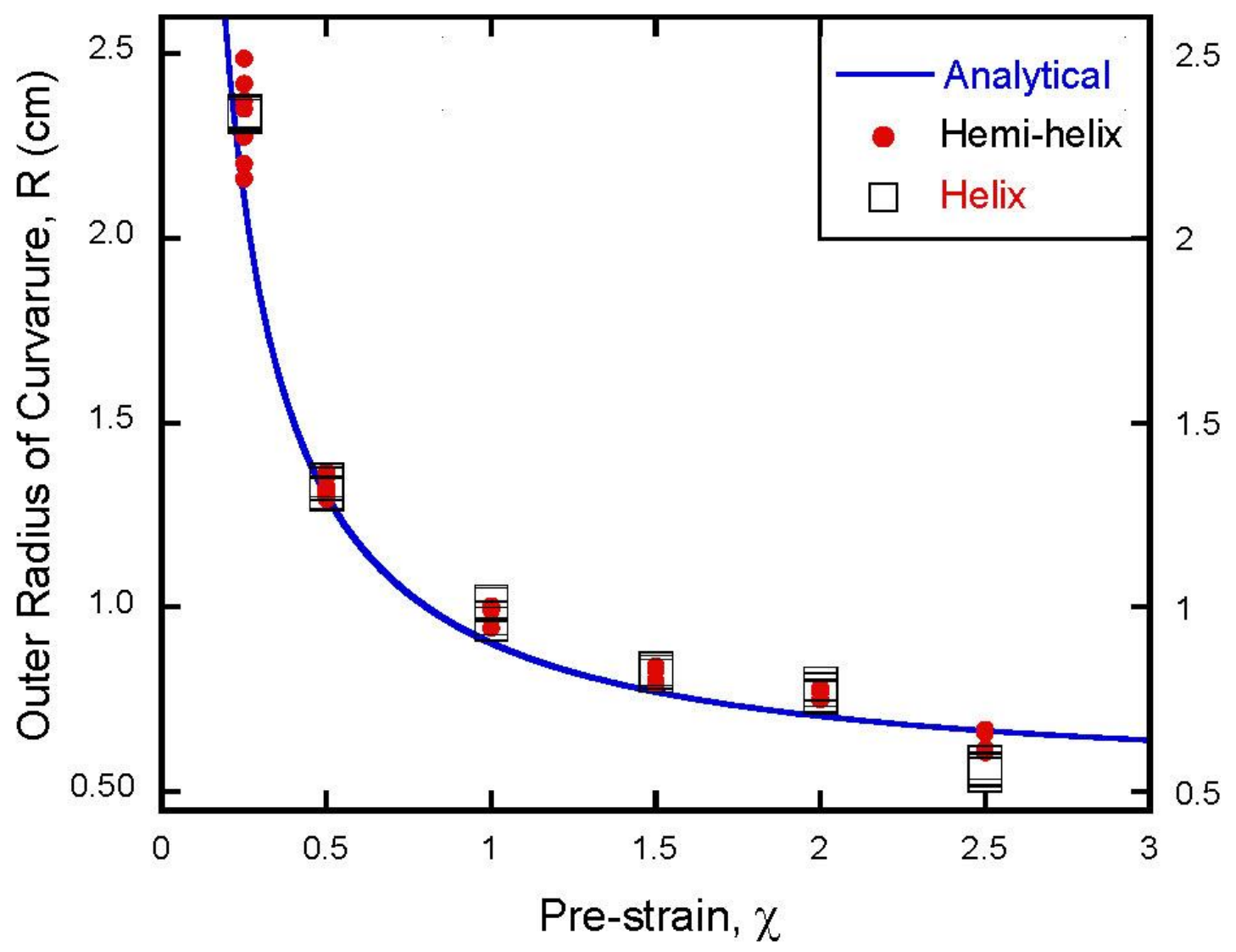

Figure 4. Huang et al. 

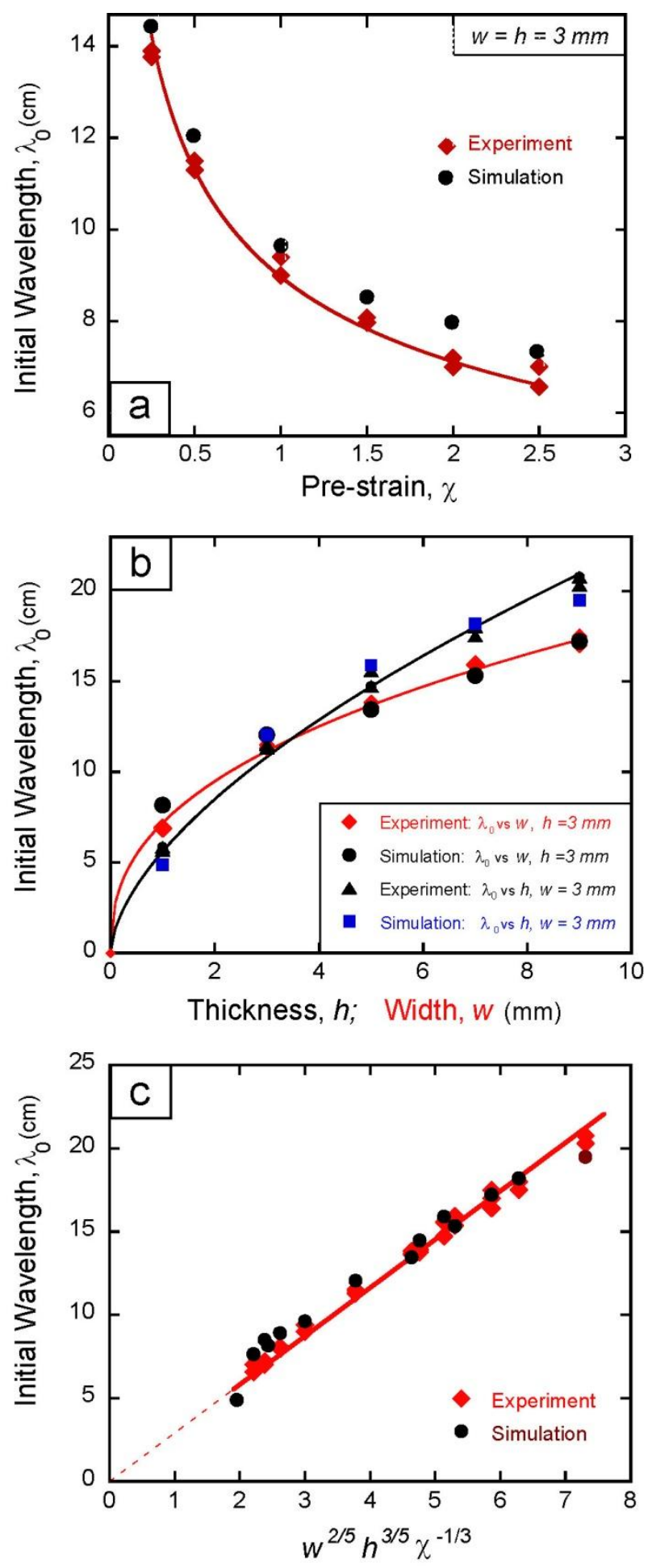

Figure 5. Huang et al. 


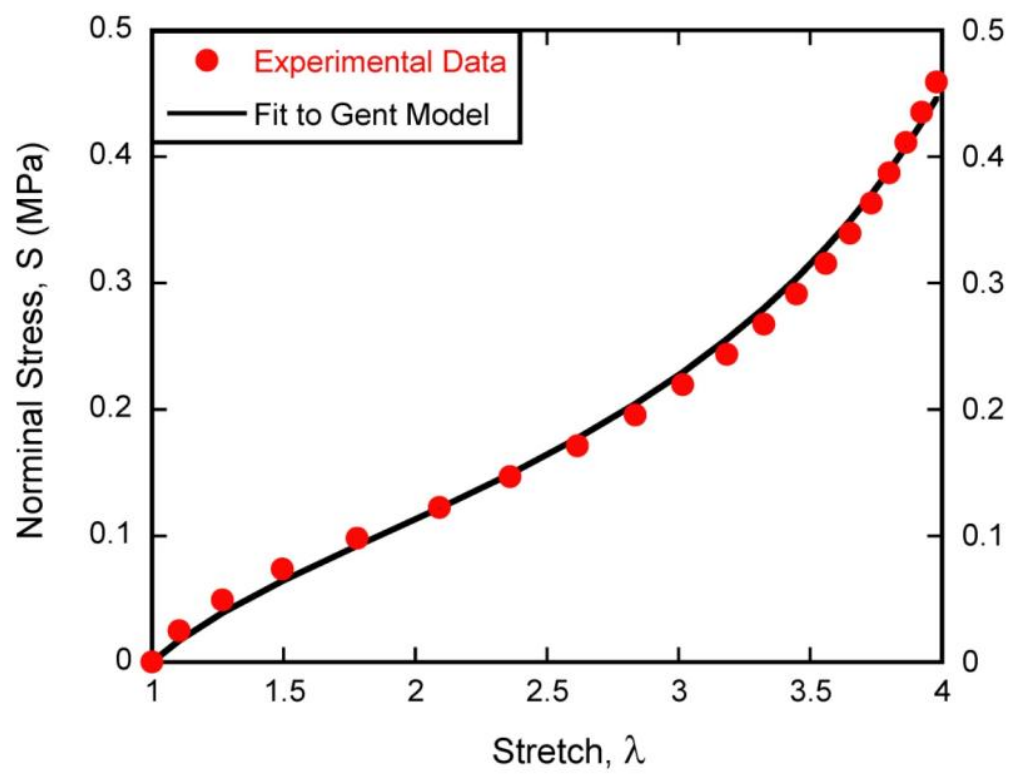

Figure 6. Huang et al.

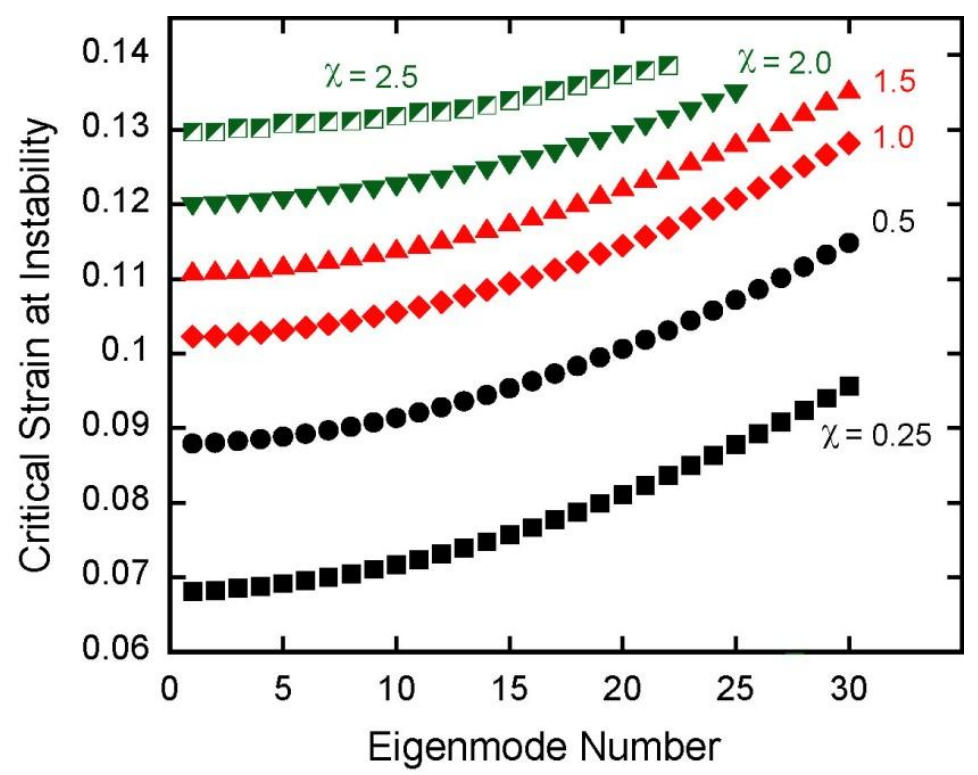

Figure 7. Huang et al. 
(a)

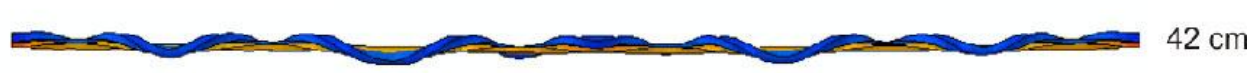

von Mises stress
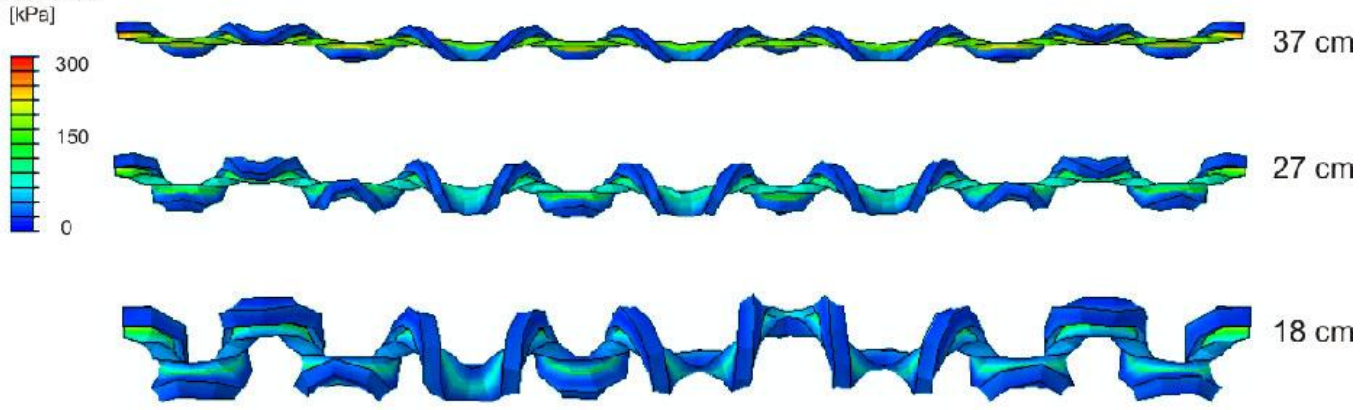

(b)

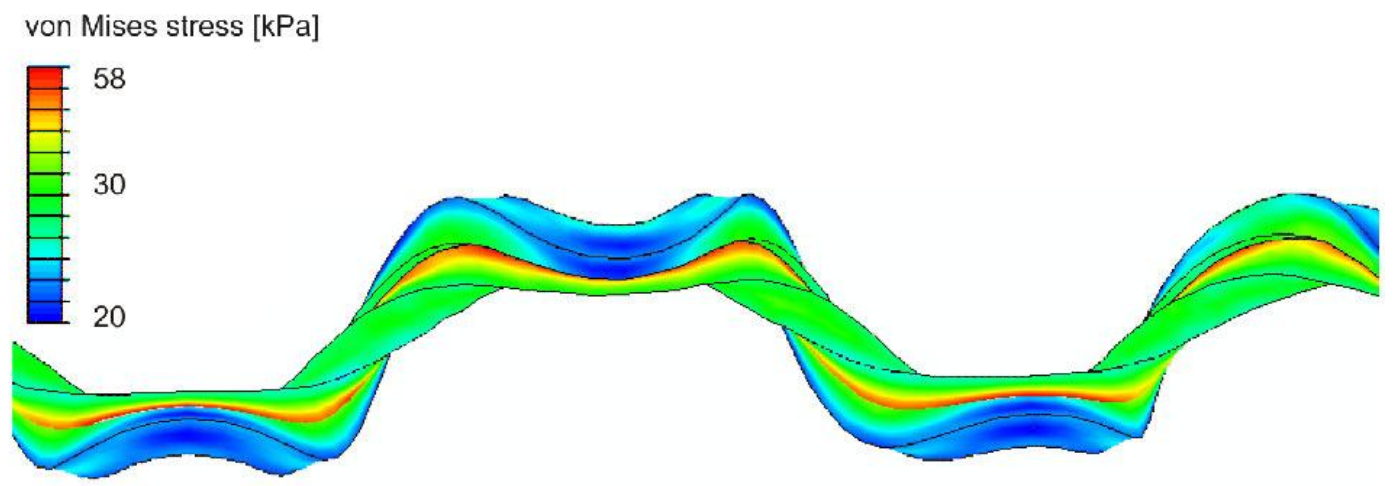

Figure 8. Huang et al. 


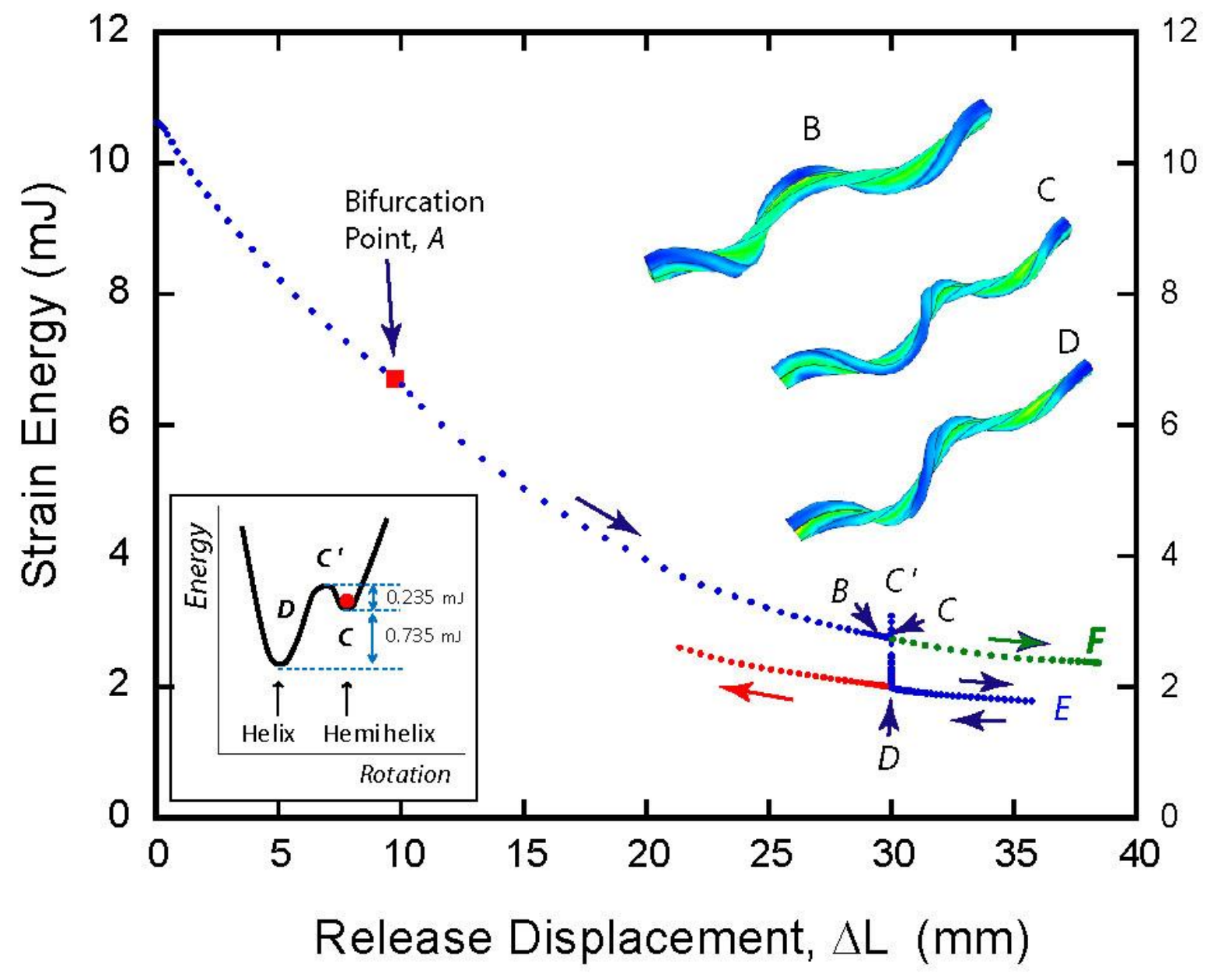

Figure 9. Huang et al. 


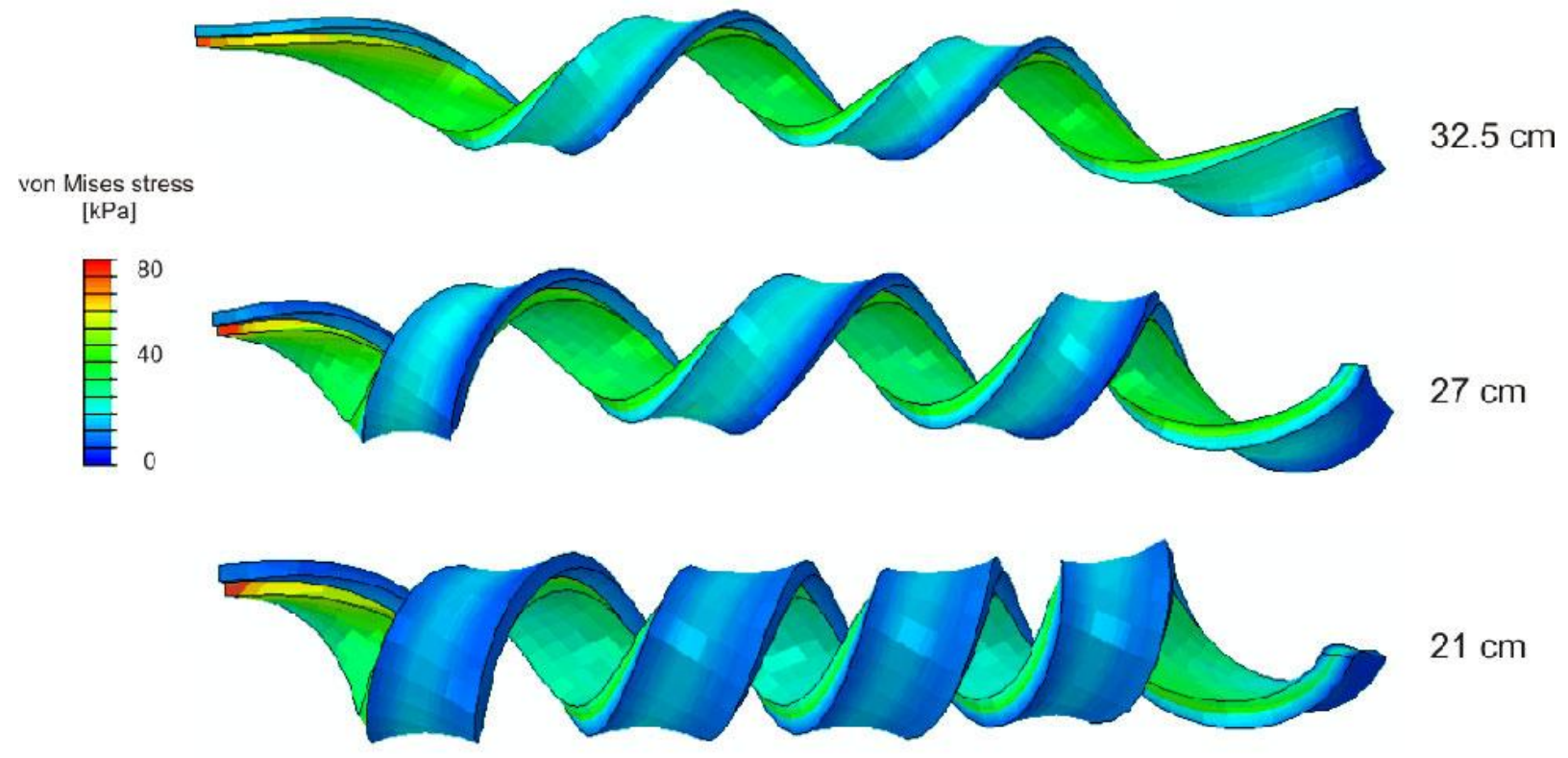

Figure 10. Huang et al. 